\title{
REAL-TIME ENVIRONMENTAL SENSORS TO IMPROVE HEALTH IN THE SENSING CITY
}

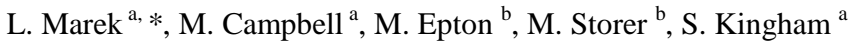 \\ ${ }^{a}$ Department of Geography, University of Canterbury, Private Bag 4800, Christchurch, New Zealand - (lukas.marek, \\ malcolm.campbell, simon.kingham)@ canterbury.ac.nz \\ ${ }^{\mathrm{b}}$ Canterbury Respiratory Research Group, Respiratory Services, Canterbury District Health Board, Private Bag 4710, Christchurch, \\ New Zealand - (michael.epton, malina.storer)@ cdhb.health.nz
}

Theme Sessions, ThS17

KEY WORDS: GIS and Health, COPD, Smart Cities, Environmental Monitoring, Air Pollution

\begin{abstract}
:
The opportunity of an emerging smart city in post-disaster Christchurch has been explored as a way to improve the quality of life of people suffering Chronic Obstructive Pulmonary Disease (COPD), which is a progressive disease that affects respiratory function. It affects 1 in 15 New Zealanders and is the 4th largest cause of death, with significant costs to the health system. While, cigarette smoking is the leading cause of COPD, long-term exposure to other lung irritants, such as air pollution, chemical fumes, or dust can also cause and exacerbate it. Currently, we do know little what happens to the patients with COPD after they leave a doctor's care. By learning more about patients' movements in space and time, we can better understand the impacts of both the environment and personal mobility on the disease. This research is studying patients with COPD by using GPS-enabled smartphones, combined with the data about their spatiotemporal movements and information about their actual usage of medication in near real-time. We measure environmental data in the city, including air pollution, humidity and temperature and how this may subsequently be associated with COPD symptoms. In addition to the existing air quality monitoring network, to improve the spatial scale of our analysis, we deployed a series of low-cost Internet of Things (IoT) air quality sensors as well. The study demonstrates how health devices, smartphones and IoT sensors are becoming a part of a new health data ecosystem and how their usage could provide information about high-risk health hotspots, which, in the longer term, could lead to improvement in the quality of life for patients with COPD.
\end{abstract}

\section{INTRODUCTION}

\subsection{Christchurch and its opportunity}

Imagine you had the chance to rebuild a city. Imagine you were able to get continuous flows of data about all sorts of things i.e. traffic or air pollution, from technology built into that city. Then, let's link that data to data about your health conditions. This is the idea behind Sensing City, Christchurch, New Zealand (NZ) (Fig. 1). The initiative was created in recognition of unique opportunities presented by the rebuild of Christchurch, following the devastating earthquakes of 2010 and 2011. The Christchurch context is unique for a number of reasons. Firstly, the city's status as a post-disaster environment with a highly damaged physical infrastructure where up to $80 \%$ of the buildings in the Central Business District (CBD) have (or will be) demolished (Marquis et al., 2015). Secondly, a population of about 367,800 people (Statistics New Zealand, 2015), much smaller than other metropolitan megacities that have entered into the 'smart city' space (e.g. New York, London, Rio de Janeiro), makes it a novel environment in which to "develop" the idea of the smart city; it is easier to disseminate and gain acceptance for novel ideas than in other urban contexts globally. During the redesign and rebuid of the CBD the opportunity to develop a 'smarter city' has presented itself via the incorporation of a range of sensors into the physical infrastructure of the area. As this platform develops and matures, linking this to health information becomes not only possible but vital.

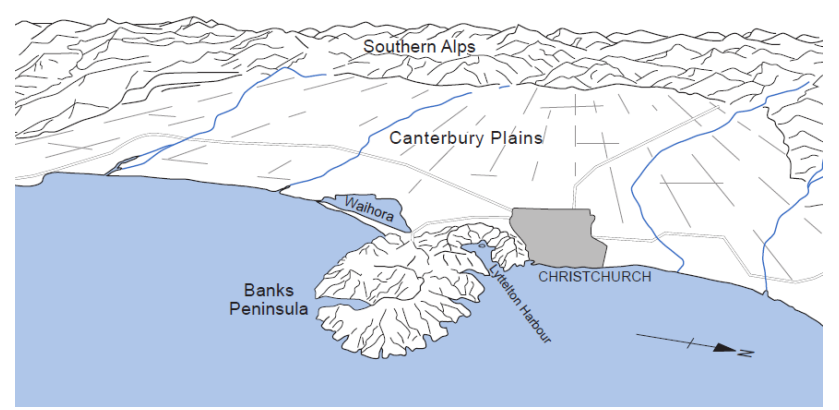

Figure 1 The view of the Christchurch (Sturman and SpronkenSmith, 2001)

\subsection{The (air)polluted city}

The general perception of New Zealand is as a country with an unspoiled nature, without significant environmental threats. The reality (mainly in the vicinity of major cities) shows a slightly different picture. Besides natural hazards, or water quality issues (CERA, 2012), significant air pollution has effects health outcomes in New Zealand (Kuschel et al., 2012). As stated by Spronken-Smith et al. (2002), Christchurch has had a wintertime air pollution problem for much of the last century. The main cause of high air pollution is the interaction of three

\footnotetext{
* Corresponding author
} 
factors (1) the use of solid fuel for domestic home heating; (2) settled anticyclonic conditions in winter which result in the temperature inversion; and (3) local topographical influences, which cause the convergence of cold air drainage over the city (Spronken-Smith et al., 2002). Figure 2 shows the annual $\mathrm{PM}_{10}$ exposure map of Christchurch in 2005. According to previous research up to $80 \%$ of the $\mathrm{PM}_{10}$ is generated by domestic woodburning open fires and enclosed wood-burners (Scott and Gunatilaka, 2004; Smithson, 2011), while the rest comes from traffic and industrial sources. Before 2010, Christchurch experienced an average of around 30-35 days when the National Environmental Standard threshold $\left(50 \mu \mathrm{g} \cdot \mathrm{m}^{-3}\right)$ was exceeded (Appelhans et al., 2010). However, the number of $\mathrm{PM}_{10}$ exceedances each year in Christchurch had reduced by 2010 and the target is 3 days by 1 September 2016 and 1 day by 1 September 2020 (Environment Canterbury, 2014). This air quality affects the health of citizens, aggravating respiratory or cardiovascular diseases (Epton et al., 2008; McGowan, 2000; McGowan et al., 2002).

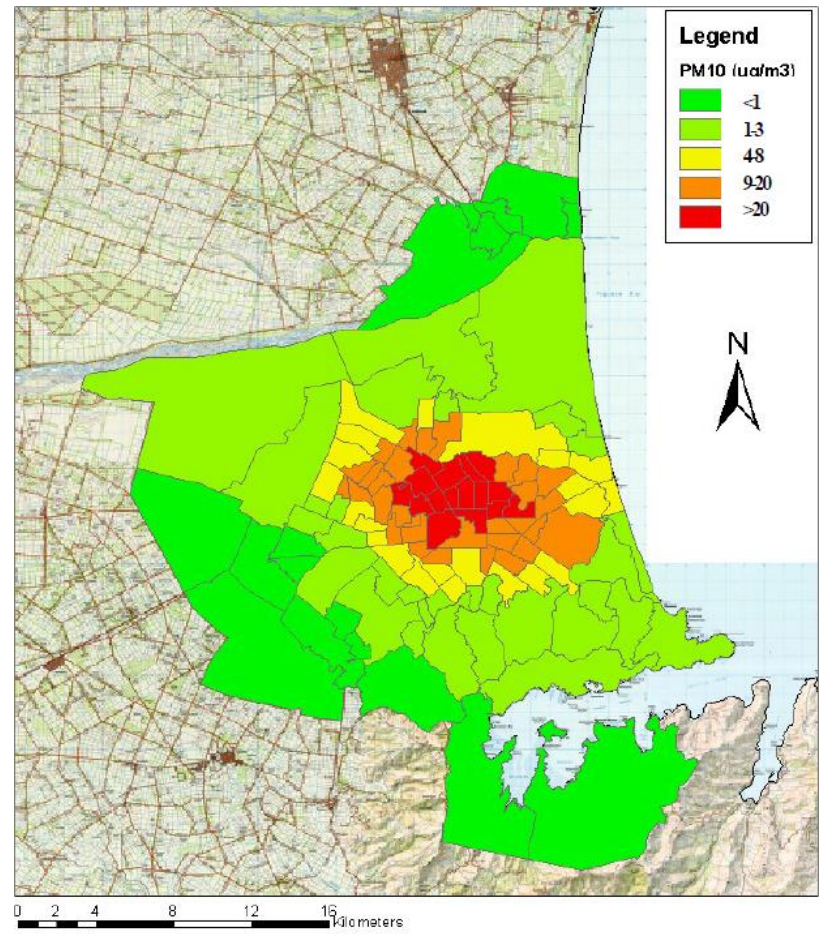

Figure 2. Annual $\mathrm{PM}_{10}$ exposure map of Christchurch in 2005 (Fisher et al., 2005)

\subsection{The City and its Health}

COPD (Chronic Obstructive Pulmonary Disease) is a progressive lung disease that makes it hard to breathe, leading to daya to day impairment. In addition, patients with COPD experience flare-ups - "exacerbations", often casued by infection, but also caused by high levels of other iriitants such as pollution.. Cigarette smoking is the leading cause of COPD. In NZ most people who have COPD smoke or are former smokers. Treatments and lifestyle changes can improve quality of life, increase activity, and slow the progress of the disease. Long-term exposure to other lung irritants, such as air pollution, chemical fumes, or dust - may also contribute to COPD. We are measuring data about what is happening in the city and its surroundings and how this may be associated with COPD. The areas of Christchurch from where patients with COPD were admitted to hospital in 2010 are depicted on the Fig. 3. We are storing the flow of the data; analysing the data and then visualising the results. We want to share not only the visualisation and analysis but the data as well. Early monitoring of air quality (for example $\mathrm{PM}_{10}$ - particulate matter $<10 \mu \mathrm{m}$ ) and meteorological data is on-going and will expand further (e.g. traffic movement data).

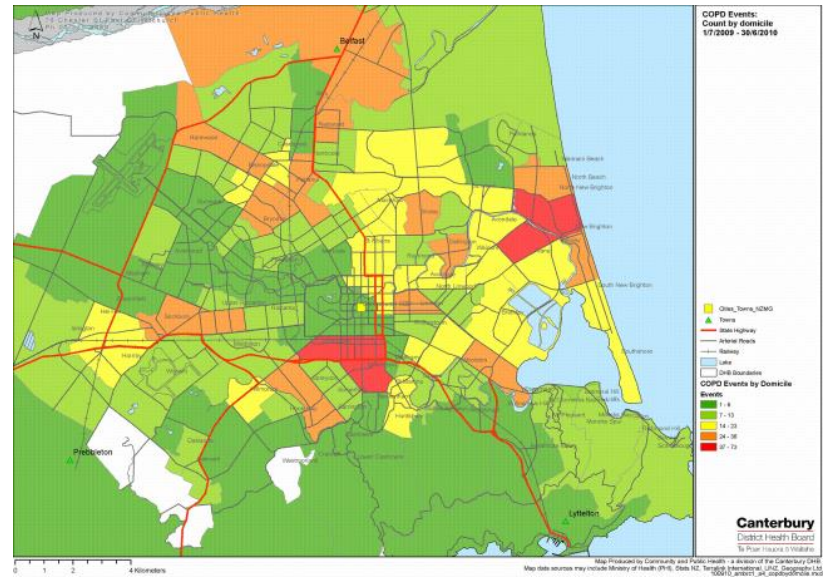

Figure 3. COPD admission events in Christchurch (NZ) (CDHB, 2010)

\section{MEASURING THE AIR QUALITY IN CHRISTCHURCH}

Currently the government body responsible for air monitoring is Environment Canterbury (ECAN) who has made some of the processed as well as raw air quality data collected publically available. But although this data is a regularly updated stream of information about meteorological and air quality conditions in the Canterbury region, this is not strictly speaking, 'smart data' or 'big data'; rather it is regularly updated data that is accessible on the web. The current system of air quality monitoring includes two sites in Woolston and St. Albans suburbs of Christchurch. This means that dispersion modelling, in other words, estimation or interpolation, is used to fill the gaps in data at present. The dispersion modelling of $\mathrm{PM}_{10}$ and other substances in the Christchurch area have been subject of a number of local studies (e.g. Appelhans, 2010; Appelhans et al., 2010; Zawar-Reza et al., 2005). In this case, we are aiming to analyse and provide the air pollution data at a finer spatial resolution in order to fill gaps around monitoring air quality while keeping the network at a lower cost.

\subsection{Extending the current network}

In order to extend the existing, air quality monitoring network, four precision dust mote devices were deployed (manufacturer?). Three of them are placed at carefully selected sites around the inner city while a fourth was co-located with one of ECAN monitoring stations for data quality evaluation. These devices together with ECAN monitoring sites provide the high precision air quality data. We have also deployed low cost Internet of Things (IoT) air quality sensors (Air Quality Eggs (AQEggs)) that further increase of the spatial coverage with minimal additional costs but, with a corresponding compromise in accuracy (see Fig. 4 for the location of individual devices). Moreover, in the near future the current network is going to be expanded with the deployment of another 20 high precision sensors that will allow unprecedented meteorological and exposure modelling in the city of Christchurch. 
The IoT sensors also support the role of the community engagement since they are able to be placed in schools, libraries or citizens homes. The air quality data can be provided to all citizens and interested sites in their primary form as well as in the form of processed data as maps and tables. In later stages of the research, the collected data will be interconnected with spatio-temporal movements of people suffering from COPD.

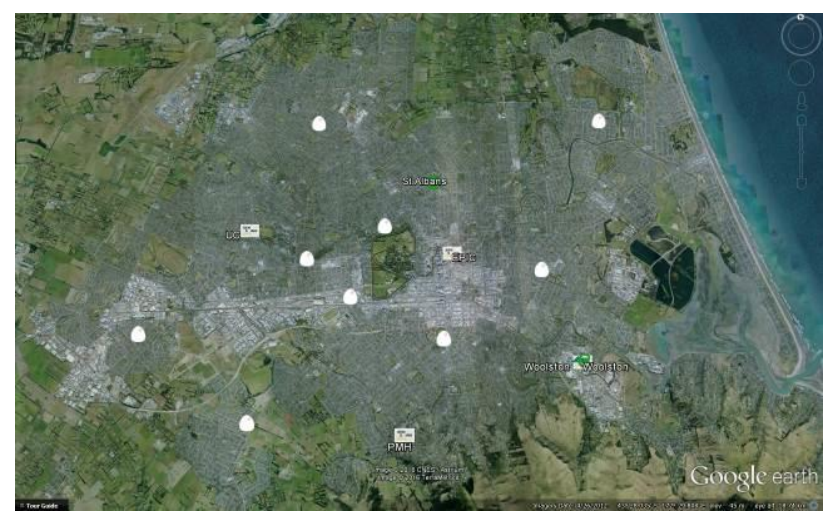

Figure 4. Sensors setup locations proposal in Christchurch (green sign - ECAN sites; white rectangle - high quality dust motes; white eggs - AQEggs)

2.1.1 Data precision issues: There are several problems with the utilisation of air quality data from different sources and different types of sensors if the system aims to offer the data to the public in a coherent way. Firstly, the quality of the monitoring devices needs to be evaluated. The internal consistency of all devices should be robust. The accuracy, precision, and homogeneity of the measurements from professional monitoring sites (ECAN, dust motes) are assured. However data generated by the low-cost platforms (as AQEggs) are not intended to replace well-calibrated official monitoring stations, but rather augment the density of the total monitoring network with citizen sensors (Demuth et al., 2013). We analysed the variability between individual AQEggs then compared them to the ECAN measurement station. The evaluation of variability and sensitivity detected similar trends in all examined AQEggs. However, the sensitivity (and precision) of individual sensors within AQEggs varied significantly (see Fig. 5), which might cause inaccuracies when the deployed.

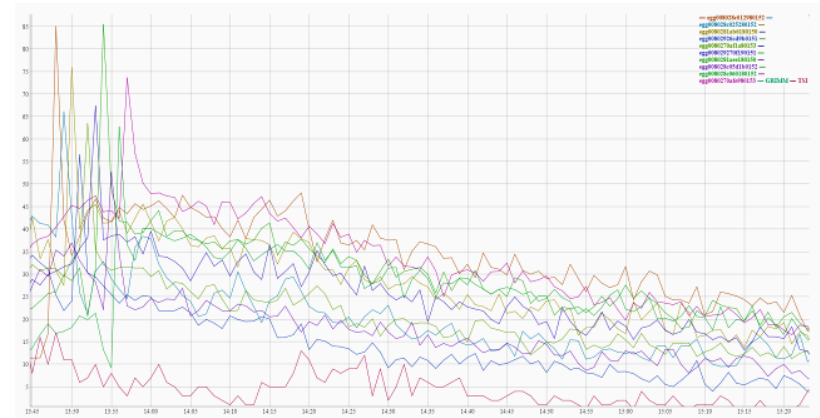

Figure 5. Comparison of sensitivity and precision of individual AQEggs measuring $\mathrm{PM}_{10}$ (1-minute aggregation) $\left[\mu \mathrm{g} \cdot \mathrm{m}^{-3}\right]$ during an indoor testing setup
Another issue is that the data from an 'official' source has nuances associated with its published data. It is not necessarily obvious to an outside observer that there have been scientific choices made about which data to publish before it appears in the public domain. However, data from the low cost sensors publish raw data without being cleaned.

\section{REAL-TIME MONITORING OF PATIENTS}

COPD affects 1 in 15 New Zealanders over 45 years and is the $4^{\text {th }}$ largest cause of death, costing the health system significant time and money. Currently, we do not know much about what happens to these patients after they leave a doctor's care. Inhalers are prescribed in COPD to help reduce breathlessness ("relievers"), and there is marked variability between individuals and for the same individual at different times, in reliever inhaler use. by better understanding the the spatiotemporal routine of patients we gain knowledge about how this disease affects them. When this information is connected to air quality data then we are able to estimate population exposure and vulnerability more accurately. This research examines patients with COPD by using smart medical devices, smart phones and smart sensors. We are about to know a patient's daily movements in close to real time in space and time. Longer term interventions based on this information could lead to dramatic changes in these patients' quality of life. Understanding everyday experiences in space and time will aid better understanding of this disease in a smart city.

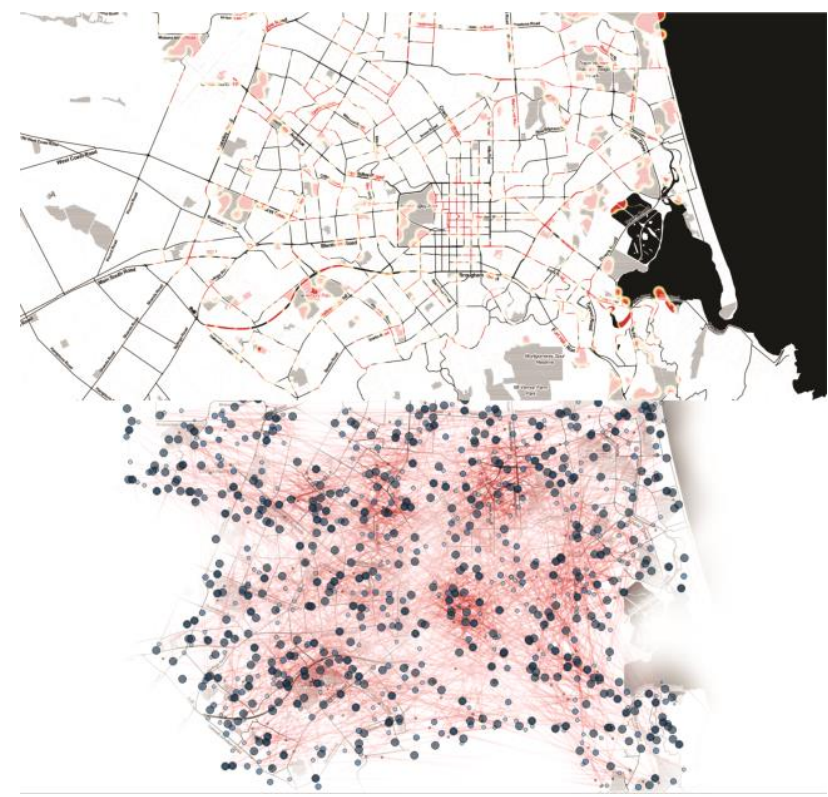

Figure 6. Spatial pattern of activities tracked by volunteers (top - red areas indicate more frequently visited areas of the city). Individual tracks and usage of smart medical devices (bottom).

For the pilot study, we track the volunteer patients using the GeoHealth Tracker application for Android smart phones that has been developed by University of Canterbury. The application is not available from Google Play Store, but Android smartphones have the ability to accept the installation of any "app" developed by a third-party and distributed outside of the official store - a process commonly known as "sideloading" (Glasgow et al., 2014). The application runs in the background of patients smart phones (in case they do not have one we are providing the smart phone) and it collects the location of the smart phone in 15-minute intervals. Following 
versions of the application may have more advanced intelligent sampling time algorithm selector based on the actual change of smart phone's position. GPS is used primarily for the capture of the position. When the GPS signal is not available, the application can also determine the location of the device (and user) by the usage of information from cell towers or Wi-Fi signal. These methods decrease the precision of captured data, however the information about the precision of the location is recorded and it can be taken into account during the analysis. By using the paired data of movements groups of patients and their reliever usage, we can start to understand the relationship between spatial and temporal clusters of inhaler usage and pollution and other environmental exposures. In order to protect privacy of individual patients, we are only using cluster approaches.

Figure 6 visualises such an example of the data pairing. The top part of the picture depicts the spatial pattern of daily activities of tracked volunteers. Red areas are visited by them on regular basis while the black areas rather sparsely. One can see the centres of volunteers' activities that are located in the southwestern suburb of the city, the city centre itself and the main streets connecting these centres. The bottom part of the picture links the flowlines of patients' daily movement with the data from smart medical devices. Using the opacity, the map focuses on places where these devices were used more frequently while the other areas of the city are rather side-lined. These visualisations are static, they describes overall spatial pattern of both, the people's movement and actual usage of their medication. One can clearly see the most frequently visited areas of the city and the activity routines of volunteers. However, there are no other data directly linked to the system, so the knowledge gained from the data pairing is very general.

\section{LINKING THE DATA: SYSTEM OUTLOOK}

For the pilot project, the data from the smart phone apps are combined with the interpolated surfaces of $\mathrm{PM}_{10}$ exposure. The daily and weekly regimes as well as seasonality of the $\mathrm{PM}_{10}$ concentrations were described by number of studies (e.g. Appelhans, 2010; Appelhans et al., 2010; Zawar-Reza et al., 2005), so the applicable model core has been already created but needs to be adjusted in order to get results in a finer spatial scale. Based on these findings and real-time monitoring data, the $\mathrm{PM}_{10}$ exposure maps can be generated. The $\mathrm{PM}_{10}$ exposure surfaces can be used as a basis for both visual analytics and spatio-temporal analysis. Figure 7 depicts generated exposure surface and use it as base information.

Using the smart phone app, we are able to track the spatiotemporal pattern of patients' schedules together with the mapping of their medication usage. The location data are then combined with the modelled exposure. By that means, we are able to approximate exposure to $\mathrm{PM}_{10}$ at any point in space and time during daily activities. This data can then be collated for groups of individuals to look for clusters of exposure and effect as it is depicted by Figure 7. The lighter are the flow lines in the figure, the less exposed were the volunteers. And on the contrary, the darker are the flow lines, the higher $\mathrm{PM}_{10}$ concentrations appeared in the area of volunteers' movements. Currently, in the initial phase of the pilot project, a limited amount of data has been streamed into data silos and system itself is not fully linked and centralised, so the data analysis and following geovisualisation is not generated online in (near)realtime automatically. The proposed system will consist of (1) the people component that collects information about the volunteers and their medical condition; (2) the environmental component collecting the air quality data, meteorological data etc.; (3) the analysing and forecasting component that will evaluate the current conditions and analyse them in order to identify the situation that might be affecting the health of people of COPD; (4) the visualisation component depicting the collected data in (near) real time together with a short-time forecasts; (5) the publishing component that is supposed to provide raw and analysed data in the machine readable format to interested users (with appropriate privacy constraints and "blurring" to protect confidentiality).

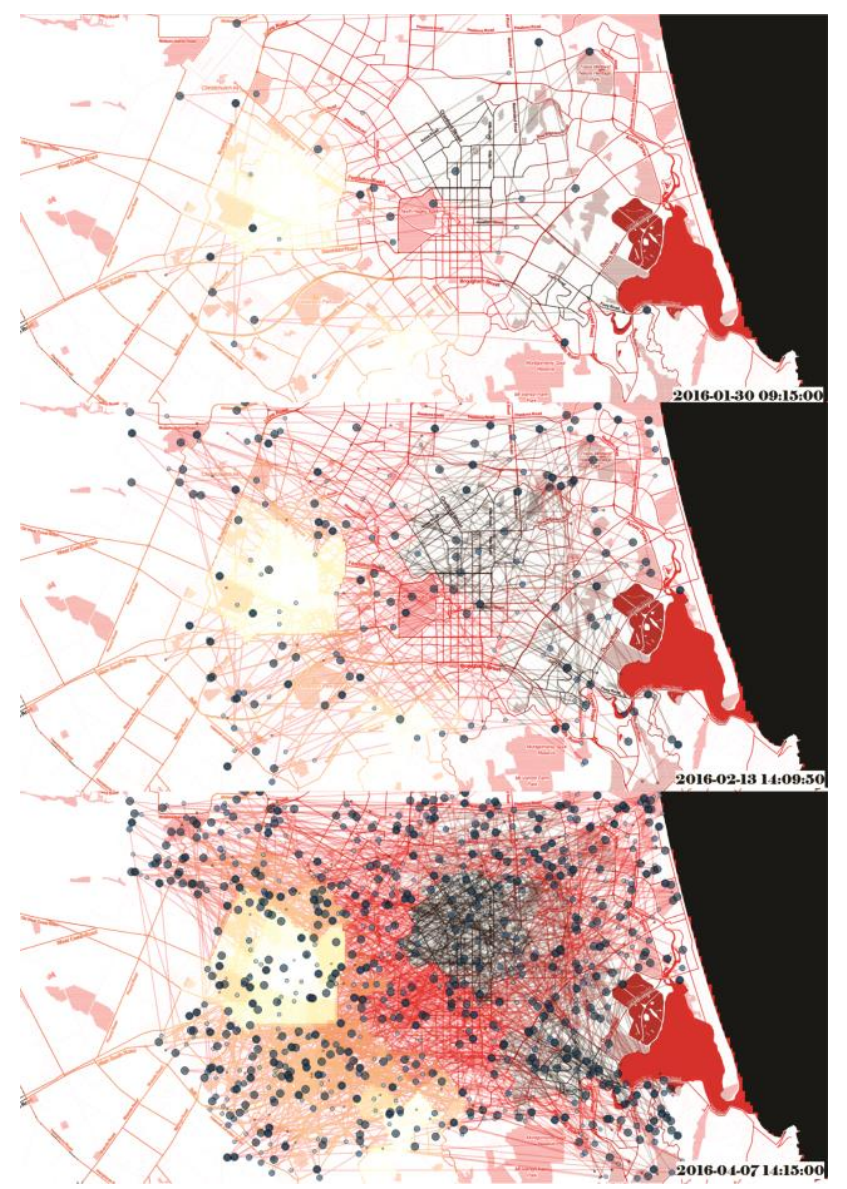

Figure 7. Time slices of volunteers' flowlines combined with $\mathrm{PM}_{10}$ exposure surface and the usage of their medical devices

\section{PRIVACY AND ETHICS}

A major challenge for implementing smart city health projects that inform individuals, public health personnel and city planners and administrators is the need to protect individual patient confidentiality whilst maximising availability of data for analysis and dissemination. We have explored this topic with focus groups of patients with COPD in Christchurch, and their conclusions are that availability of data collected from individuals, if appropriately stored, with blurring of disseminated data to prevent individual identification, is justified because of the potential benefits to individuals and groups of patients with COPD. They also highlighted the importance that the technology we use to collect data from individual volunteers is easy to use and acceptable.

The privacy and confidentiality of personal data is the crucial task that directly influences the data providers and analytics. The use of patient identifiers such and address could be used to identify patients and Point locations obtained from geocoding or GPS can be used in the same way (Waller and Gotway, 2004). This same data confidentiality is also the reason for the 
strain between the usefulness of the analysis in the local scale and the protection of the individual person privacy (Marek et al., 2013). For these reasons the volunteers' individual data are secured by the password and encrypting system which is only accessible by the research team. We do not aim to disseminate the data in raw way any further but we want to study the anonymised dataset in a long term. All of these activities have been approved by appropriate local ethics committees and have been the subject of extensive consultation.

\section{DISCUSSION AND CONCLUSIONS}

This pilot project demonstrates that real-time data collected from volunteers with long-standing disease can be linked to time and motion data, and environmental data such as air pollution, to attempt to link, model and disseminate this data for public good. Although the presented pilot study uses the COPD patients as its core subject, the principles and methodology can be easily transferred to any health subject of interest.

As these systems and technologies develop, it is important to retain awareness of the privacy issues associated with such powerful tools. In addition, we need to develop business models and agreements to allow such datasets to be most effectively used, without excessive concern about intellectual property and "ownership". At the core of this, we need to remember that the "ownership" of time and space data generated by smart devices belongs to the volunteers.

In order to protect privacy of volunteers and the data confidentiality we used simulated tracking data throughout this paper.

\section{ACKNOWLEDGEMENTS (OPTIONAL)}

This research is supported by the Cooperative Research Centre for Spatial Information (CRCSI), whose activities are funded by the Australian Commonwealth's Cooperative Research Centres Programme (P4.49) as well as by Future Position X, Sweden.

\section{REFERENCES}

Appelhans, T., 2010. A climatology of particulate pollution in Christchurch. University of Canterbury.

Appelhans, T., Sturman, A.P., Zawar-Reza, P., 2010. Modelling emission trends from non-constant time series of PM10 concentrations in Christchurch, New Zealand. Int. J. Environ. Pollut. 43, 354-363.

Canterbury District Health Board, 2010. COPD Events: Counts by domicile 1/7/2009-30/6/2010.

CERA: Canterbury Earthquake Recovery Authority [WWW Document], 2012. URL http://cera.govt.nz/recoverystrategy/natural-environment

Demuth, D., Nüst, D., Bröring, A., Pebesma, E., 2013. The AirQuality SenseBox. Geophys. Res. Abstr. 15.

Environment Canterbury, 2014. Air quality in the Canterbury region. Environment Canterbury Environmental Snapshot Report. Christchurch, New Zealand.

Epton, M.J., Dawson, R.D., Brooks, W.M., Kingham, S., Aberkane, T., Cavanagh, J.-A.E., Frampton, C.M., Hewitt, T., Cook, J.M., McLeod, S., McCartin, F., Trought, K., Brown, L., 2008. The effect of ambient air pollution on respiratory health of school children: a panel study. Environ. Health 7, 16.
Fisher, G., Kjellstrom, T., Woodward, A., Hales, S., Town, I., Sturman, A., Kingham, S., 2005. Health and Air Pollution in New Zealand: Christchurch Pilot Study.

Glasgow, M.L., Rudra, C.B., Yoo, E.-H., Demirbas, M., Merriman, J., Nayak, P., Crabtree-Ide, C., Szpiro, A. a, Rudra, A., Wactawski-Wende, J., Mu, L., 2014. Using smartphones to collect time-activity data for long-term personal-level air pollution exposure assessment. J. Expo. Sci. Environ. Epidemiol. 1-9.

Kuschel, G., Metcalfe, J., Wilton, E., Guria, J., Hales, S., Rolfe, K., Woodward, A., 2012. Updated Health and Air Pollution in New Zealand Study - Volume 1: Summary Report.

Marek, L., Dvorský, J., Pászto, V., Pavel, T., Vávra, A., 2013. Health Datasets in Spatial Analyses: What We Want, What We Get and What We Can Use, in: Recent Advances in Geodesy and Geomatics Engineering. WSEAS Press, Antalya, pp. 240246.

Marquis, F., Kim, J.J., Elwood, K.J., Chang, S.E., 2015. Understanding post-earthquake decisions on multi-storey concrete buildings in Christchurch, New Zealand. Bull. Earthq. Eng.

McGowan, J.A., 2000. Effects of particulate air pollution on cardiorespiratory admissions in Christchurch, NZ. University of Canterbury.

McGowan, J.A., Hider, P.N., Chacko, E., Town, G.I., 2002. Particulate air pollution and hospital admissions in Christchurch, New Zealand. Aust. N. Z. J. Public Health 26, 23-29.

Scott, A., Gunatilaka, M., 2004. Christchurch inventory of emissions to air. R04/03. Christchurch, New Zealand.

Smithson, J., 2011. Inventory of emissions to air in Christchurch, R11/17. Christchurch, New Zealand.

Spronken-Smith, R.A., Sturman, A.P., Wilton, E. V, 2002. The air pollution problem in Christchurch, New Zealand - progress and prospects. Clean Air Environ. Qual. 36, 23-29.

Statistics New Zealand, 2015. Subnational Population Estimates: At 30 June 2015 (provisional).

Sturman, A.P., Spronken-Smith, R.A. (Eds.), 2001. The Physical Environment: a New Zealand Perspective. Oxford University Press, Melbourne.

Waller, L.A., Gotway, C.A., 2004. Applied Spatial Statistics for Public Health Data. John Wiley \& Sons.

Zawar-Reza, P., Kingham, S., Pearce, J., 2005. Evaluation of a year-long dispersion modelling of PM10 using the mesoscale model TAPM for Christchurch, New Zealand. Sci. Total Environ. 349, 249-259. 